\title{
Delay analysis of multihop wireless ad hoc network using queuing network model
}

\author{
Seema Kumari, Dr.M.U.Kharat \\ PG student at G.H.R.C.E.M Pune, Wagholi Pune, Maharashra India \\ engineerseema@gmail.com \\ Prof at G.H.R.C.E.M Pune, Wagholi Pune, Maharashtra India
}

\section{ABSTRACT} mukharat@rediffmail.com

\begin{abstract}
Ad hoc wireless network is a self configurable infrastructure less network. The lack of infrastructure support in ad hoc networks makes them useful in various applications such as at the disaster site, highway, vast forest, battle field, oceans, etc. Since there is no centralized control in ad hoc network, each node can act as a source, a destination or a router. Ad hoc wireless networks therefore, experience congestion more than the wired networks, leading to jitter and high end to end delays even for moderate traffic which may lead to performance degradation. So it is crucial to analyze the factors which affect the capacity and end-to-end delay in wireless ad-hoc networks. In this paper a G/G/1queuing network model is proposed togain insights into the end-to-end delay in a multi hop wireless ad hoc networks. Queuing network model is unique as it providesclosed form expressions for average end-to-end delay in multihop wireless ad hoc networks. NS2 simulation is conducted in order to verify and compare the theoretical results.
\end{abstract}

\section{Indexing terms/Keywords}

Ad-hoc network, Capacity, Congestion, End-to-End delay, Jitter, Queuing network model.

\section{Academic Discipline And Sub-Disciplines}

Computer Network

\section{SUBJECT CLASSIFICATION}

E.g., Mathematics Subject Classification; Library of Congress Classification

\section{Council for Innovative Research}

\author{
Peer Review Research Publishing System
}

Journal: INTERNATIONAL JOURNAL OF COMPUTERS \& TECHNOLOGY

Vol 10, No 4 


\section{LITERARY SURVEY}

Previous work on the capacity of wireless ad hoc networks is found in [2], [6], [7], [11], [12], and [14]-[17]. In reference paper [17] Gupta and Kumar presented the asymptotic results for static networks, in their study they have shown that peruser network capacity is $\mathrm{O}(\mathcal{W} n)$ and hence it vanishes as the number of users $n$ increases. They have also shown that there exists a global scheduling scheme achieving $\Omega(\mathcal{W}(n$ log $n)) \quad$ for a uniform random network and random traffic pattern. It is not worthwhile that the throughput reaches zero available to each node when the number of nodes increases. In reference paper [15] the effect of mobility on the capacity of ad-hoc wireless networks was studied, in their work the authors have developed a 2-hop relay algorithm which supports the constant per-user throughput which means that throughput does not vanish when the size of the network grows. In reference paper [16] the authors examine the ad hoc forwarding and IEEE 802.11 MAC interactions and they also examine the effect on capacity for several topologies like chain, uniform lattice, single cell and random networks. They obtained an estimate of the expressions for one-hop capacity and upper bound on per-node throughput using simulated results. The trade-offs between throughput and delay is characterized by authors in reference paper [11]. In their work they characterized the delay and throughput for static and mobile nodes in wireless networks. They have shown that for a network with static nodes the average delay and throughput are related by $D(n)=\Theta(n T(n))$, where $D(n)$ and $T(n)$ are the average end-to-end delay and throughput respectively. The size of the packet scales with throughput according to the network model [4]. Under these assumptions delay is simply proportional to the average number of hops i.e. there is no delay due to queuing. The queuing network model proposed in this paper takes into account queuing delay at the source and intermediate nodes.

Queuing networks is an effective system modeling method which is widely used in the modeling and performance analysis of various computer and communication systems. The queuing models are generally used to analyze the queuing behavior mathematically. In reference paper [13], a finite queuing model is proposed to evaluate the blocking probability of a packet and MAC queuing delays in a Basic Service Set. A queuing model is proposed and used in reference paper [8] for performance evaluation of WLAN which is based on the IEEE 802.11 MAC, in the presence of HTTP traffic. The authors modeled the service time of a node in wireless ad hoc network based on IEEE 802.11 MAC as a Markov modulated general arrival process and the resulting M/MMGI/1/K queuing model is used for analyzing the delay over a single hop in the wireless network in reference paper [9]. The queuing theoretic approach is used in reference paper [1] to calculate the maximum throughput, collision probability and mean packet delay for a four node elementary network with hidden nodes and the results are extended to linear wireless networks. The prior work [6]-[17] has not been extended to two dimensional wireless networks.

\section{INTRODUCTION}

Ad hoc wireless networks are becoming more and more popular nowadays. As Compare to the infrastructure wireless network set up, the wireless ad hoc network does not require router or a wireless base station for establishing a connection. This kind of topology is useful sometimes, especially in military applications and sensor networks. As ad hoc networks are self configurable each node can act as a sender, a receiver or a router. As a router, it can route the packet to the destination or next router in the route if necessary. Each node can buffer packets when the packets need to wait for transmission. Hence Ad hoc wireless networks experience congestion more than wired networks. Several recent studies have focused on characterizing the capacity and delay in wireless ad hoc networks. In most of these studies, there is no delay due to queuing, but if it is assumed that the packet size is constant and the packet delay is defined as time taken by a packet to reach the destination after it arrives at the source, the queuing delays would occur at the source and intermediate nodes. In this paper an analytical model based on open G/G/1 queuing networks is proposed for analyzing the average end-to-end delay in multi hop wireless ad hoc networks.

\section{QUEUING NETWORK MODEL}

Open $\mathrm{G} / \mathrm{G} / 1$ queuing network model is used to model a multi hop wireless ad hoc network. G/G/1 describes a single server system where interarrival times and service times both have general distribution. The queuing network model is developed by using the network model and traffic model. The network model may be described as follows: There are $n+1$ nodes in the network which are distributed uniformly and independently over a torus of unit area. It is assumed that each node have an equal transmission range, denoted by $r(n)$. Let the distance between nodes $i$ and $j$ is denoted by $r_{i j}$. Nodes $i$ and $j$ are known as neighbors if they can directly communicate with each other, i.e. if $r_{i j} \leq r(n)$. Let the set of nodes that are neighbors of node $\mathrm{i}$ is denoted by $\mathrm{N}(\mathrm{i})$. Let $\mathrm{W}$ bits per second denotes the transmission range of each node. A special case of the Protocol Model of interference [17] is used, it may be described as follows: If node $i$ transmits to node $j$ then the transmission will be successful only if

(a) $\quad r_{i j} \leq r(n)$ and

(b) $\quad r_{k j}>r(n)$ 
for every other node $\mathrm{k} \neq \mathrm{i}, \mathrm{j}$ that transmits simultaneously with node $\mathrm{i}$.

The traffic model may be described as follows: Each node in the network could be a sender, receiver and/or relay of packets. The packet generation rate of each node is $\lambda$ packets/s. The packet generation process is Poisson process. Each packet size is constant and it equals $L$ bits. When a packet is received by a node from any of its neighbors, it either forwards the packet to its neighbors with probability $(1-p(n))$ or absorbs the packet with probability $p(n)[2]$. Probability $p(n)$ is known as absorption probability[2]. The probability $(1-p(n))$ is known as forwarding probability. This traffic model is used to characterize the effect of locality of traffic on the average end-to-end delay in a multi hop wireless ad hoc network.

A multi hop wireless ad hoc network modeled as a queuing network model is shown in Fig. 1. The nodes of the ad hoc network are represented as stations of the queuing network in Fig. 1(a) and the forwarding probabilities are represented by $\mathrm{P}_{\mathrm{ij}}$. Figure $1(\mathrm{~b})$ shows the representation of a single node in ad hoc network as a station in queuing network model. This queuing network model is used to mathematically analyze the average end-to-end delay.

$$
P_{51}(n)
$$

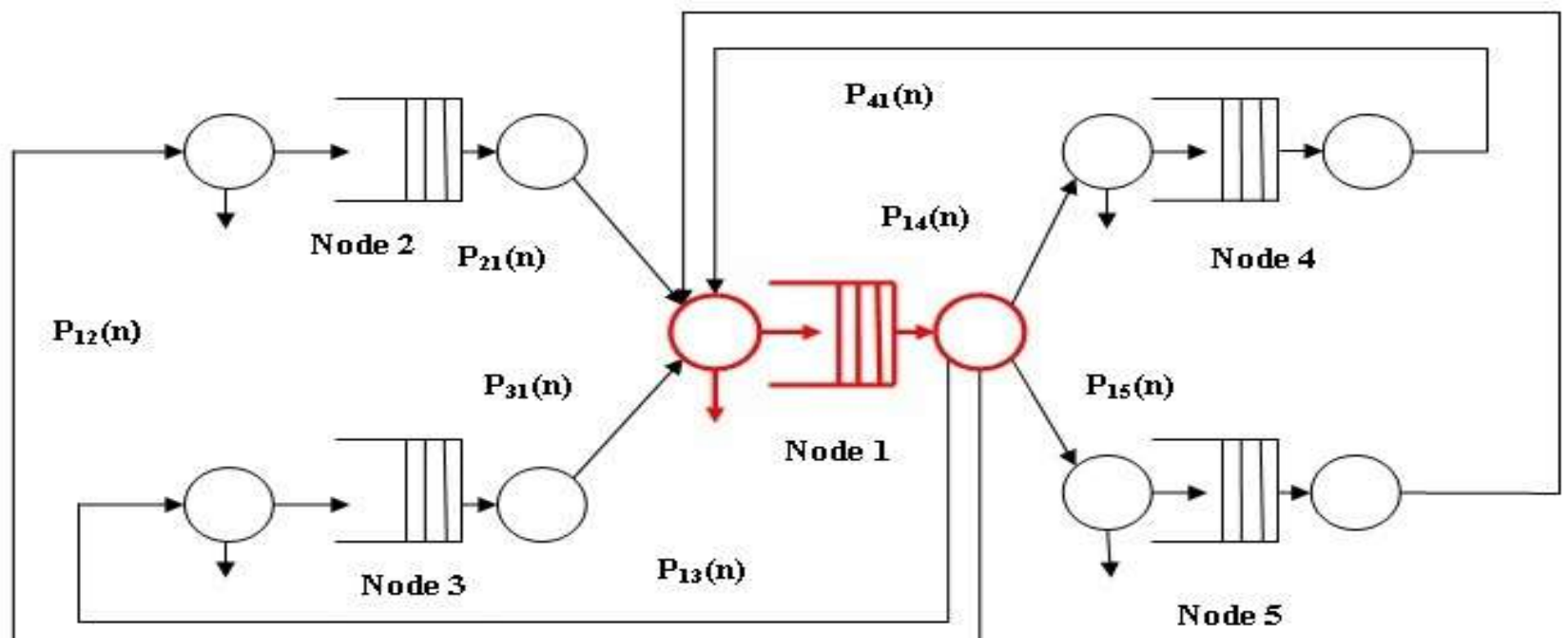

(a)

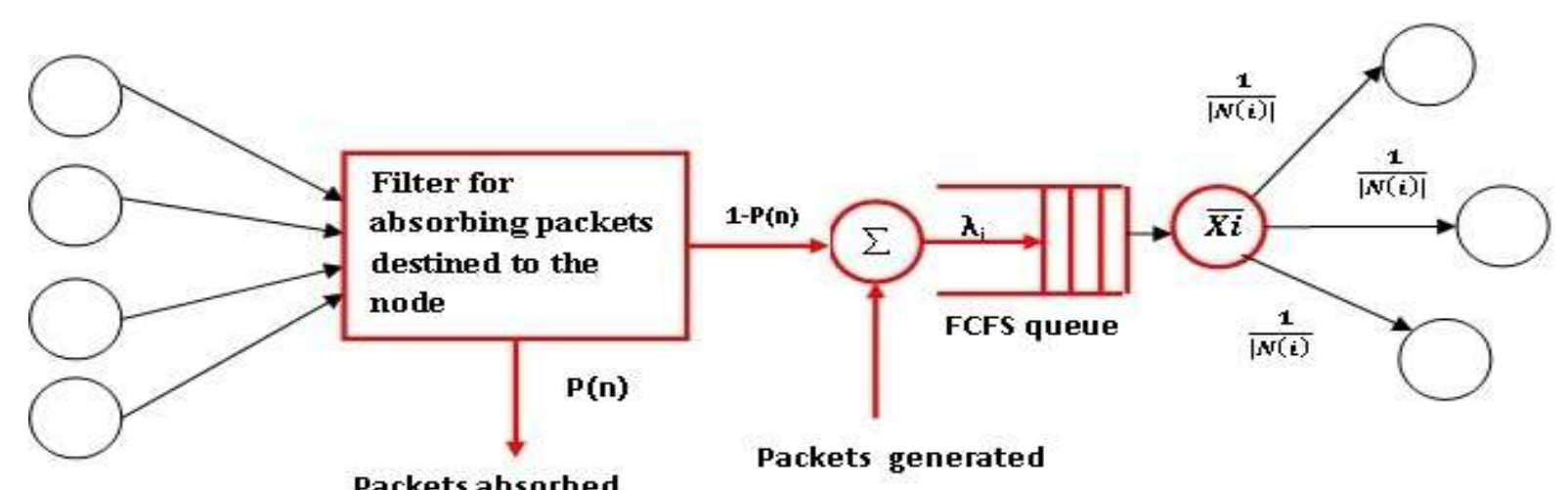

(b)

Figure 1.Queuing network model for multi-hop wireless ad hoc network. (a) Representation of multi-hop wireless ad hoc network as a queuing network. (b) Representation of a node of multi-hop wireless ad hoc network.

\section{Parameters of the queuing network model}

The important parameters of the queuing network model of multi-hop wireless networks are as follows:

The expected probability that node i forwards a packet to node $\mathrm{j}$, denoted by $\mathrm{p}_{\mathrm{ij}}$ is given as follows 


$$
\overline{p_{1 y}}(\mathrm{n})= \begin{cases}\frac{1-p(n)}{n}\left(1-(1-A(n))^{n}\right) & i \neq j \\ 0 & i=j\end{cases}
$$

(1)

The expected visit ratio of node $\mathrm{i}$, which is denoted by $\overline{e_{2}}$ is given as follows

$$
\overline{e_{u}}=\frac{1}{(n+1) p(n)} \forall \mathbf{i}
$$

The effective packet arrival rate at node $i$, denoted by $\lambda_{i}$ is given by

$$
\lambda_{i=} \lambda / p(n)
$$

The detailed description of these results are presented in reference paper [2].

\section{DELAY ANALYSIS}

The end-to-end delay in a wireless network is equal to the sum of queuing and transmission delays at source and intermediate nodes. The queuing network model shown in Fig. 1(a) and 1(b) is used to mathematically analyze the end-toend delay. The detailed proofs are presented in reference paper [2]. The mean and second moments of the service time of nodes are evaluated using the random access MAC model and the result or average end-to-end delay in multi-hop wireless ad hoc networks, denoted by $D(n)$ is given by

$$
\mathrm{D}(\mathrm{n})=\frac{\rho_{\mathbf{i}}}{\lambda \cdot(1-\hat{\rho})}
$$

\section{Where}

$\rho_{\mathrm{i}}=\lambda_{i} \bar{X}_{1}$, utilization factor of station $\mathrm{i}$

$$
\begin{aligned}
& \bar{X}_{i}=\frac{\frac{1}{\xi}+\frac{L}{W}}{1-4 n A(n) \lambda_{i} \frac{L}{W}} . \\
& \hat{\rho}_{i}=\exp \left(-\frac{2\left(1-\rho_{i}\right)}{c_{A i}^{2} \cdot \rho_{i}+c_{B i}^{2}}\right) .
\end{aligned}
$$

$\mathrm{C}^{2} \mathrm{Ai}: \mathrm{SCV}$ (squared coefficient of variance) of station i.

$\mathrm{C}^{2}{ }_{\mathrm{Bi}}$ : SCV of service time of station $\mathrm{i}$.

Although the result obtained as above provides a closed form expression of end-to-end delay, it does not provide direct intuition into how delay scales with various network parameters. This is because of the complex dependence of utilization factor on various network parameters. In this paper it is simplified for an asymptotic case with network size tends to infinity and the packet generation rate $(\lambda)$ tends to the maximum achievable per-node throughput of the network. Various approximations are used to simplify the above expressions and finally a closed form bound on the average end-to-end delay is obtained as follows:

$\bar{D} \geq \frac{1+\alpha}{2} \frac{L}{W} \frac{\beta}{1-\beta} \sqrt{\frac{n}{\log n}}$

Or

$$
\bar{D}=\Omega\left(\frac{\beta}{1-\beta} \sqrt{\frac{n}{\log n}}\right)
$$

where

$\bar{D}=$ average end to end packet delay 
$\propto=$ constant, mean distribution of back off timer is represented as a product of packet transmission time and a constant $\alpha>0$ i.e $\frac{1}{\xi}=\alpha \frac{L}{W}$

$\beta=$ constant, the packet generation rate $\lambda$ is represented as product of maximum achievable throughput and a constant $\beta$ $>0$, i.e. $\lambda=\beta \frac{W / L}{4 \pi \sqrt{n \log n}}, \beta>0$

$\mathrm{n}=$ network size i.e very large ( $\mathrm{n}$ tends to infinity).

These results present more valuable insight into how delay scales with various network parameters than the result presented in equation (4). For fixed network size, the average end-to-end delay scales $\frac{\lambda}{\lambda \max -\lambda} \mathrm{k}$ with $\mathrm{k}$. For a fixed packet generation rate, the average end-to-end delay scales as $\sqrt{\frac{n}{\log n}}$.

\section{SIMULATION AND RESULTS}

The NS2 simulation is as follows. A network consists of $n$ nodes which are distributed uniformly over $500 \mathrm{~m} * 500 \mathrm{~m}$ area. On each node an exponential traffic source is attached to produce the packets of length of 1000 bytes at rate of $\lambda / s e c o n d$. Each node chooses a random destination and the traffic is routed to the destination using DSDV routing protocol. In this network, UDP protocol is used as transport layer protocol. The IEEE 802.11 MAC and free space propagation models are used for the simulations. Table 1 shows the details of the configurations.

Table 1. Simulation setup

\begin{tabular}{|l|l|}
\hline Parameter & Value \\
\hline Transmission range $\mathrm{r}(\mathrm{n})$ & $30-150$ \\
\hline Topology size & $500 \times 500$ \\
\hline Number of nodes $(\mathrm{n})$ & $100-700$ \\
\hline Simulation Time & $1000 \mathrm{sec}$ \\
\hline Traffic type & Constant bit rate \\
\hline Packet generation rate $(\lambda)$ & $0.2-0.8$ \\
\hline Packet size $(\mathrm{L})$ & $1000 \mathrm{bits}$ \\
\hline Transmission rate $(\mathrm{W})$ & $1 \mathrm{Mbps}$ \\
\hline Routing Protocol & DSDV \\
\hline MAC & 802.11 \\
\hline Queue & DropTail \\
\hline Propagation models & Freespace \\
\hline
\end{tabular}

In order to validate simulation results with the analytical results the values of the parameters of the analytical model are set such that they are comparable to that of the simulation. Fig.2 shows the average end-to-end delay obtained from NS2 simulations as a function of number of nodes. It is observed that for higher network sizes (n) and traffic arrival rate $(\lambda)$ the simulation results differ substantially from the theoretical results. The increased deviation with increase in traffic is due to the three reasons: i) As number of nodes (n) in the network increases, the number of nodes contending for channel increases hence increasing the chances of packet collision. As the traffic rate $(\lambda)$ increases, the nodes attempt to transmit more often which increases the chances of collision between the packets. (ii) Due to formation of routing hotspots as a result of shortest path routing. (iii) Due to collision of data packets with routing control packets.

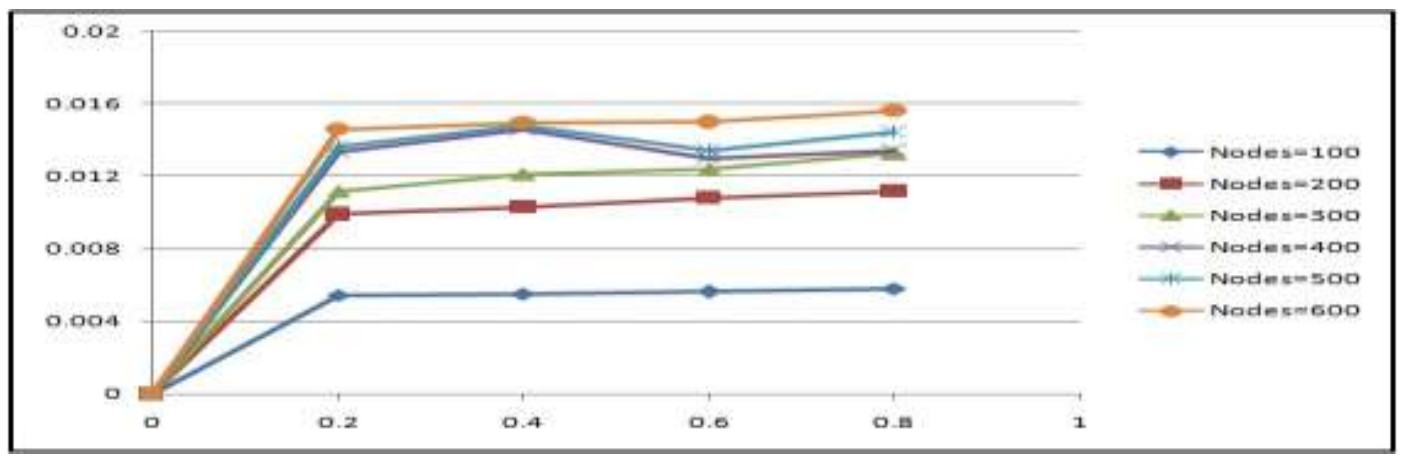

Fig2. Average end to end delay vs. number of nodes for NS2 Simulations 
Fig.3 shows the average end-to-end delay obtained from NS2 simulations as a function of packet arrival times. Here it is observed that for higher packet arrival rates and large network size the delay obtained from NS2 simulation is larger than the theoretical results. This deviation in the delay can be explained in the following manner. In shortest path routing there are many situations when some links in the network carry more traffic than the average traffic in the network. These links are known as routing hotspots. These routing hotspots become bottleneck and the packet routed through these links experience much larger delays whenever the traffic load is high. However in probabilistic routing the traffic is spread uniformly over the network and therefore the average end-to-end delay for the theoretical model increases less rapidly. Increment in the number of nodes leads to increment in the traffic which in turn leads to high delays on the bottleneck links. Another reason for the deviation in the delay is the following. As number of nodes increases, the diameter of the network increases and the packets traverses more number of hops between a sender and receiver.

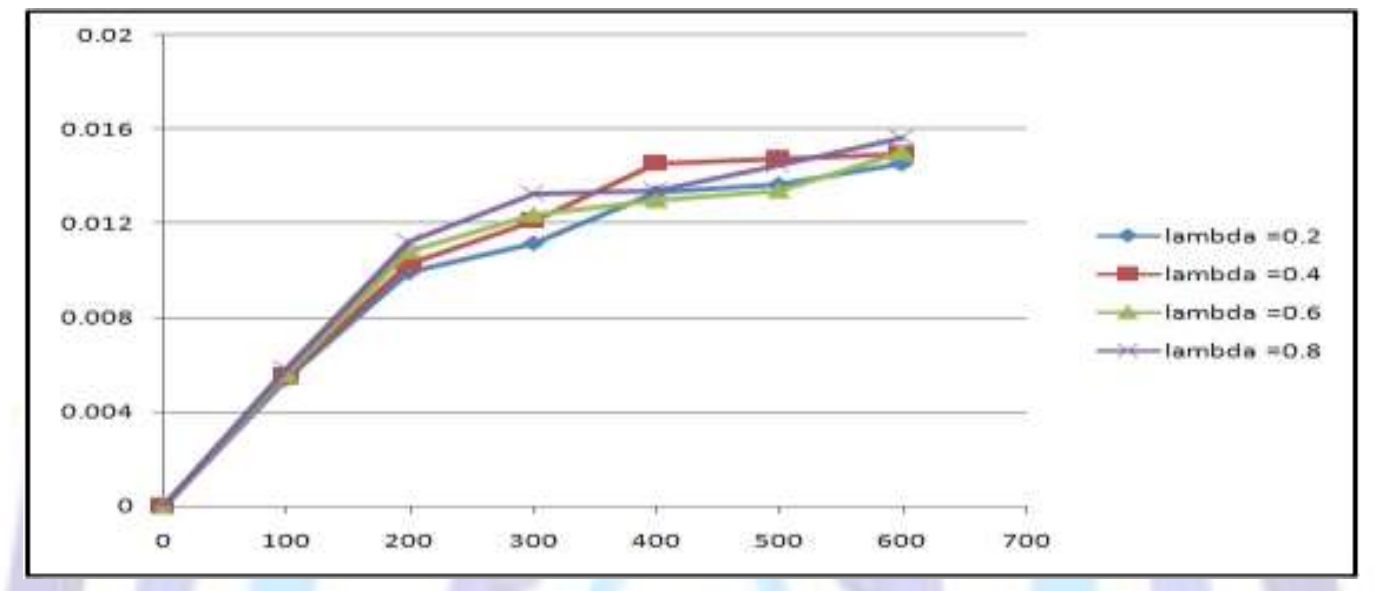

Fig 3. Average end-to-end delay vs. arrival rate for NS2 Simulations

\section{CONCLUSION}

The purpose of delay analysis presented in this paper is to capture the effect of random access MAC and queuing delays on the average end-to-end delay of multi hop wireless ad hoc networks. The analytical model presented in this paper is simple so that it is possible to obtain closed form expressions for the average end-to-end delay. The analytical results are validated with simulation results. The NS2 simulations indicate that for higher network sizes and traffic the performance of the standard wireless protocols is worse than the predicted performance using analytical model.

\section{REFERENCES}

[1]Tie QIU, Feng XIA, Lin FENG, Guowei WU, Bo JIN, Queueing theory-based path delay analysis of wireless sensor networks, AECE ,Volume 11, Number 2, 2011

[2] N.Bisnik, A.A. Abouzeid, Queuing network models for delay analysis of multihop wireless ad hoc networks, Elsevier Ad Hoc Networks 7 (2009) 79-97

[3] J.Nzouonta, T. Ott, C. Borcea, Impact of Queuing Discipline on Packet Delivery Latency in Ad Hoc Networks, Elsevier, Performance Evaluation, 2009.

[4] J. Deng, Y.S. Han, S.R. Kulkarni, Can Multiple Subchannels Improve the Delay Performance of RTS/CTS-based MAC Schemes, IEEE Trans. on Wireless Comm., Vol. 8, No. 4, April 2009.

[5] A.C. Lozano, S.R. Kulkarni, P. Viswanath, Throughput Scaling in Wireless Networks with Restricted Mobility, IEEE Trans. on Wireless Comm., Vol. 6, Feb. 2007.

[6] S. Ray, D. Starobinski, J.B. Carruthers, Performance of wireless networks with hidden nodes: A queuing-theoretic analysis, Comput. Commun. 28 (10) (2005)

[7] Michael J. Neely and Eytan Modiano,Capacity and Delay Trade-offs for Ad-Hoc Mobile Networks IEEE Trans. on Information Theory, vol. 51, no. 6, June 2005

[8] D. Miorandi, A.A. Kherani, E. Altman, A queueing model for HTTP traffic over IEEE 802.11 WLANs, in proceedings of 16th ITC specialist seminar on Performance Evaluation of Wireless and Mobile Systems, Aug.2004.

[9] M. Ozdemir, A.B. McDonald, An M/MMGI/1/K queuing model for IEEE 802.11 ad hoc networks, in proceedings of the first ACM International workshop on performance evaluation of wireless ad Hoc, sensor, and ubiquitous networks, ACM 2004. 
[10] O. Tickoo, B. Sikdar, A queueing model for finite load IEEE 802.11 random access MAC, in: Proceedings of IEEE ICC, Paris, France, June 2004.

[11] A.E. Gamal, J. Mammen, B. Prabhakar, D. Shah,Throughput-delay trade-off in wireless networks, in:Proceedings of IEEE INFOCOM , March 2004.

[12] S. Narasimhan and S. S. Kunniyur, Effect of network parameterson delay in wireless ad hoc networks, Technical Report, jun. 2004

[13] G. Zeng, H. Zhu, I. Chlamtac, A novel queueing model for 802.11 wireless LANs, in proceedings of WNCG Wireless Networking Symposium 2003

[14] N. Bansal and Z. Liu,Capacity, Mobility and Delay in Wireless Ad hoc Networks, In proceedings of IEEE INFOCOM, 2003.

[15] Mathias Grossglauser and David Tse, Mobility increases the capacity of ad-hoc wireless networks, In Proceedings of IEEE Infocom01, April 2001.

[16] J. Li, C.Blake, D. S. J. De Couto, H. Lee, and R. Morris Capacity of Ad Hoc Wireless Networks, in Proc. of IEEE Mobicom, Italy July 2001.

[17] P. Gupta, P.R. Kumar, Capacity of wireless networks, IEEE Trans. on Information Theory, March 2000.

[18] V. Bharghavan, A. Demers, S. Shenker, L. Zhang, MACAW: a media access protocol for wireless LANs, in Proceedings of the Conference on Comm. Architectures, Protocols and Applications, ACM Press 1994,

[19] P. Karn,MACA: a new channel access methods for packet radio, in: Proceedings of the Nineth Computer Networking conference, Sept.1990.

[20] K.Fall,K.Varadhan,The NS2 user manual, http://www.isi.edu/nsnam/ns/.

[21] Billy E. Gillet. Introduction to operation research

[22] Leonard Kleinrock Queueing Systems volume I: Theory

\section{Author' biography with Photo}

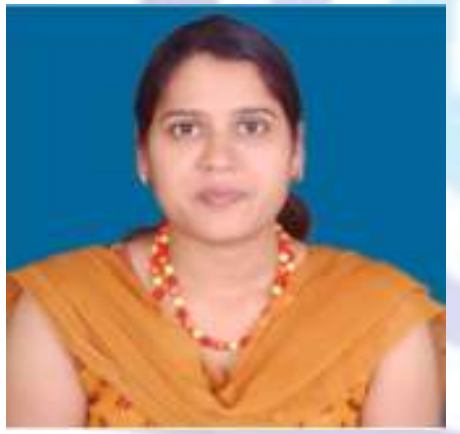

Seema Kumari received the B.Tech degree in computer science and engineering with first class from Uttar Pradesh Technical University, Lucknow, UP in 2005. She has an experience of teaching in various engineering colleges. Presently she is working as a lecturer in G.H. Raisoni Institute of engineering and Technology, Wagholi, Pune. She is pursuing M.E in computer networks from G.H.Raisoni College of Engineering and Management, Wagholi,Pune. Her areas of interest include wireless ad hoc networks.

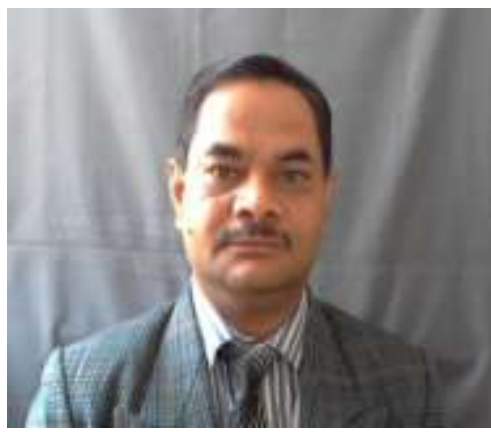

M. U. Kharat, BE, MS, Ph.D. was educated at SGBA University and Devi Ahilya University. Presently he is working at MET's IOE, Nashik, Maharashtra, India, as Professor \& Head Computer Engineering Department. He has presented papers at National and International conferences and also published papers in National and International Journals on various aspects of Computer Engineering and Networks. He has worked in various capacities in academic institutions at the level of Professor, Head of Computer Engineering Department, and Principal. His areas of interest include Digital Signal Processing, Computer Networks, and the nternet. 